\title{
Supporting Information: Resolving heterogeneous dynamics of excess protons in aqueous solution with rate theory
}

Santanu Roy, ${ }^{* \dagger}$ Gregory K. Schenter,,$\ddagger$ Joseph A. Napoli,, Marcel D. Baer, Thomas E. Markland, "and Christopher J. Mundy ${ }^{*, \ddagger \S}$

$\dagger$ Chemical Sciences Division, Oak Ridge National Laboratory, 1 Bethel Valley Rd., Oak Ridge, TN 37830, USA

$\ddagger$ Physical Sciences Division, Pacific Northwest National Laboratory, 902 Battelle Blvd, Richland, WA 99352, USA

9 Department of Chemistry, Stanford University, 333 Campus Drive Stanford, CA 94305, USA

$\S$ Affiliate Professor, Department of Chemical Engineering, University of Washington, Seattle, USA

E-mail: roys@ornl.gov; chris.mundy@pnnl.gov 


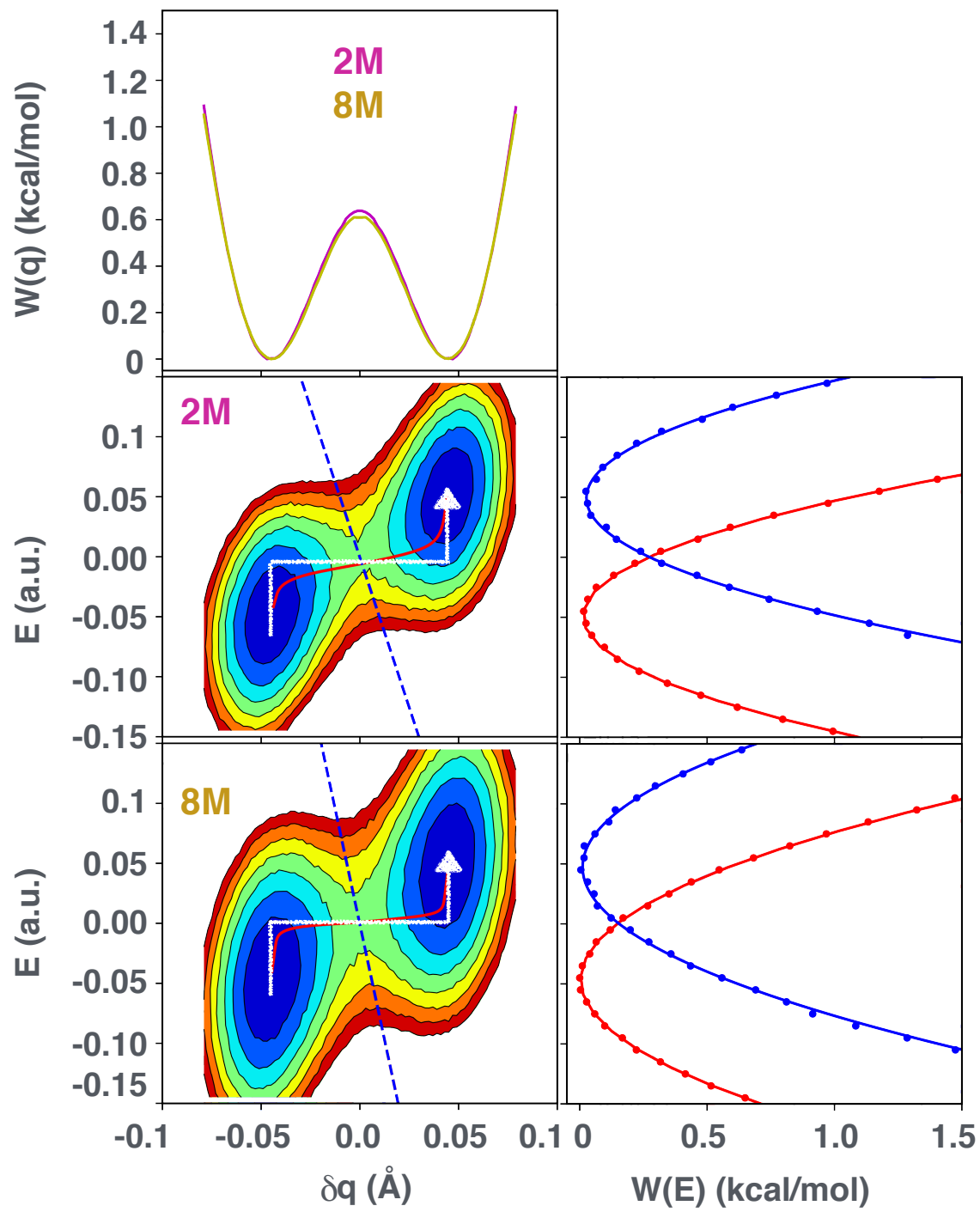

Figure S1: Comparison between the phenomena of proton transfer for $2 \mathrm{M}$ and $8 \mathrm{M} \mathrm{HCl}$ solutions: The 1D PMFs, $W(\delta q)$ (a) and $W(E)$ (c) and the 2D PMF, $W(\delta q, E)$ (b) describing that minimum free energy pathway (white, dashed) and Marcus pathway (red, solid) of proton transfer are in excellent agreement. Blue, dashed line indicate dividing surface perpendicular to the minimum free energy pathway. Dotted lines in $W(E)$ are slices (dotted line) from $W(\delta q, E)$ representing the reactant, $W_{\mathrm{R}}(E)$ and the product, $W_{\mathrm{R}}(E)$, that are fitted with parabolas (solid line) 


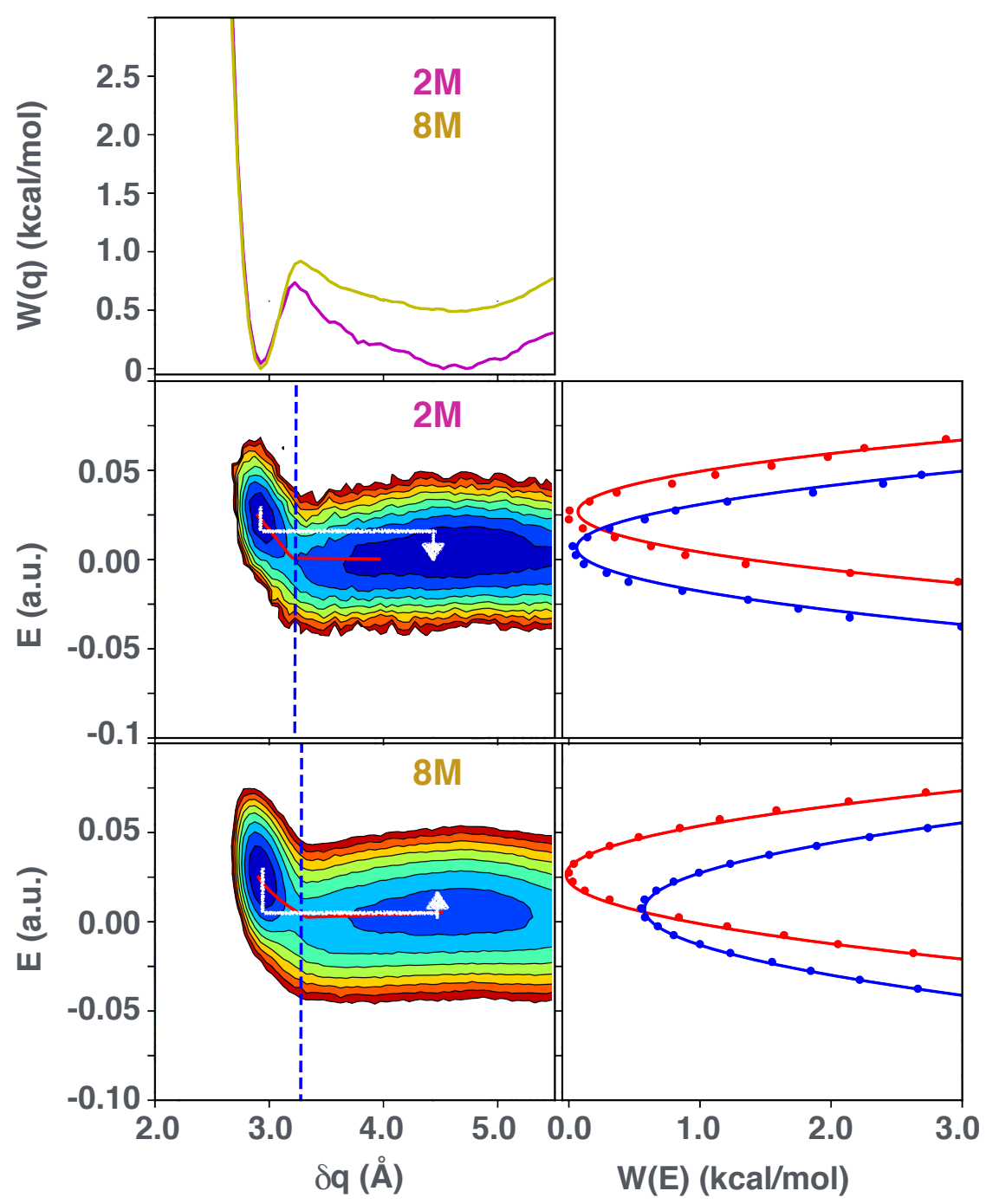

Figure S2: Comparison between the phenomena of ion pair dissociation for $2 \mathrm{M}$ and $8 \mathrm{M}$ HCl solutions: The 1D PMFs, $W(q)$ (a) and $W(E)(\mathrm{c})$ and the 2D PMF, $W(q, E)$ (b) describing that minimum free energy pathway (white, dashed) and Marcus pathway (red, solid) of proton transfer are in excellent agreement. Blue, dashed line indicate dividing surface perpendicular to the minimum free energy pathway. Dotted lines in $W(E)$ are slices (dotted line) from $W(\delta q, E)$ representing the reactant, $W_{\mathrm{R}}(E)$ and the product, $W_{\mathrm{R}}(E)$, that are fitted with parabolas (solid line) 


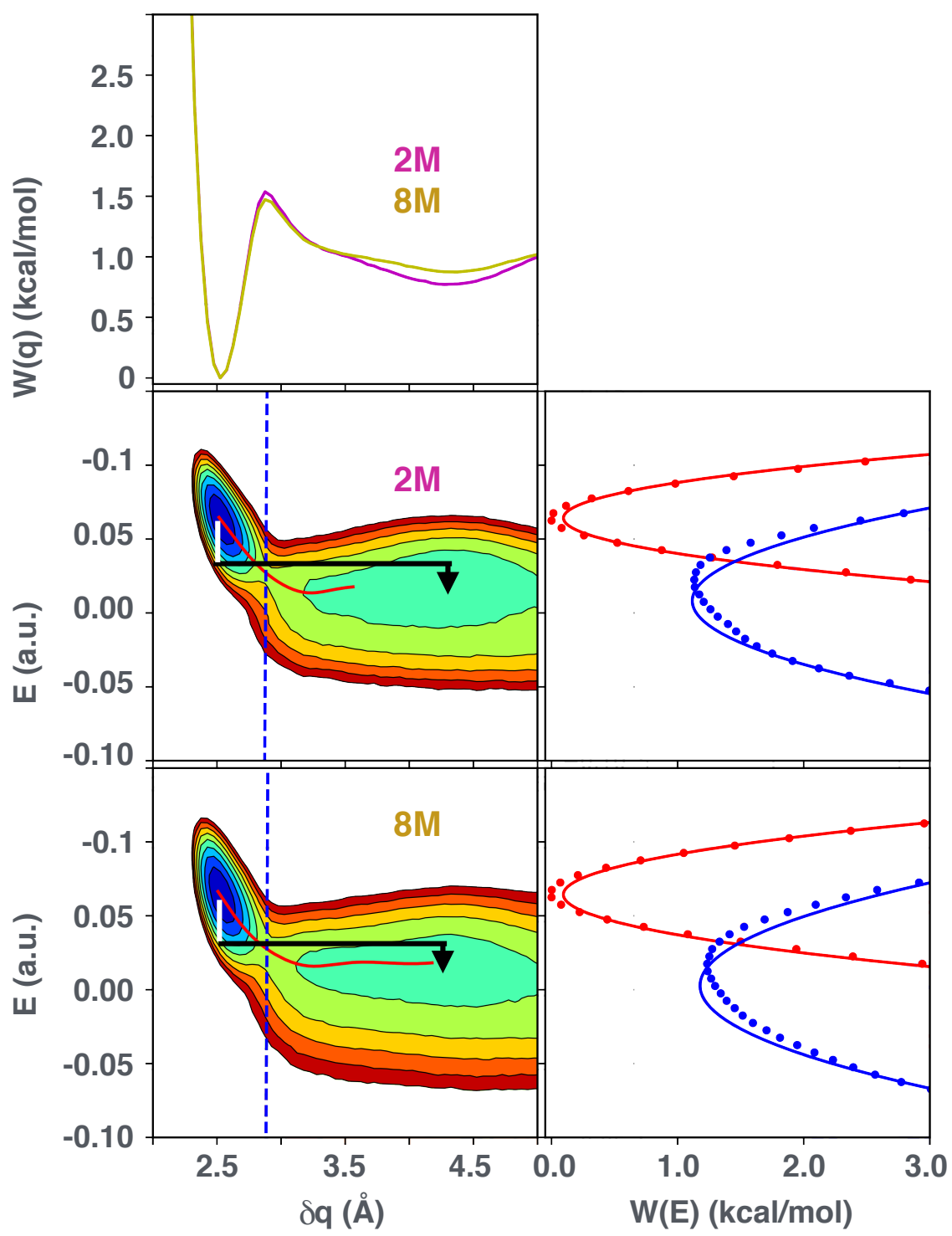

Figure S3: Comparison between the phenomena of water exchange for $2 \mathrm{M}$ and $8 \mathrm{M} \mathrm{HCl} \mathrm{solu-}$ tions: The 1D PMFs, $W(q)$ (a) and $W(E)(\mathrm{c})$ and the 2D PMF, $W(q, E)(\mathrm{b})$ describing that minimum free energy pathway (white, dashed) and Marcus pathway (red, solid) of proton transfer are in excellent agreement. Blue, dashed line indicate dividing surface perpendicular to the minimum free energy pathway. Dotted lines in $W(E)$ are slices (dotted line) from $W(\delta q, E)$ representing the reactant, $W_{\mathrm{R}}(E)$ and the product, $W_{\mathrm{R}}(E)$, that are fitted with parabolas (solid line). 

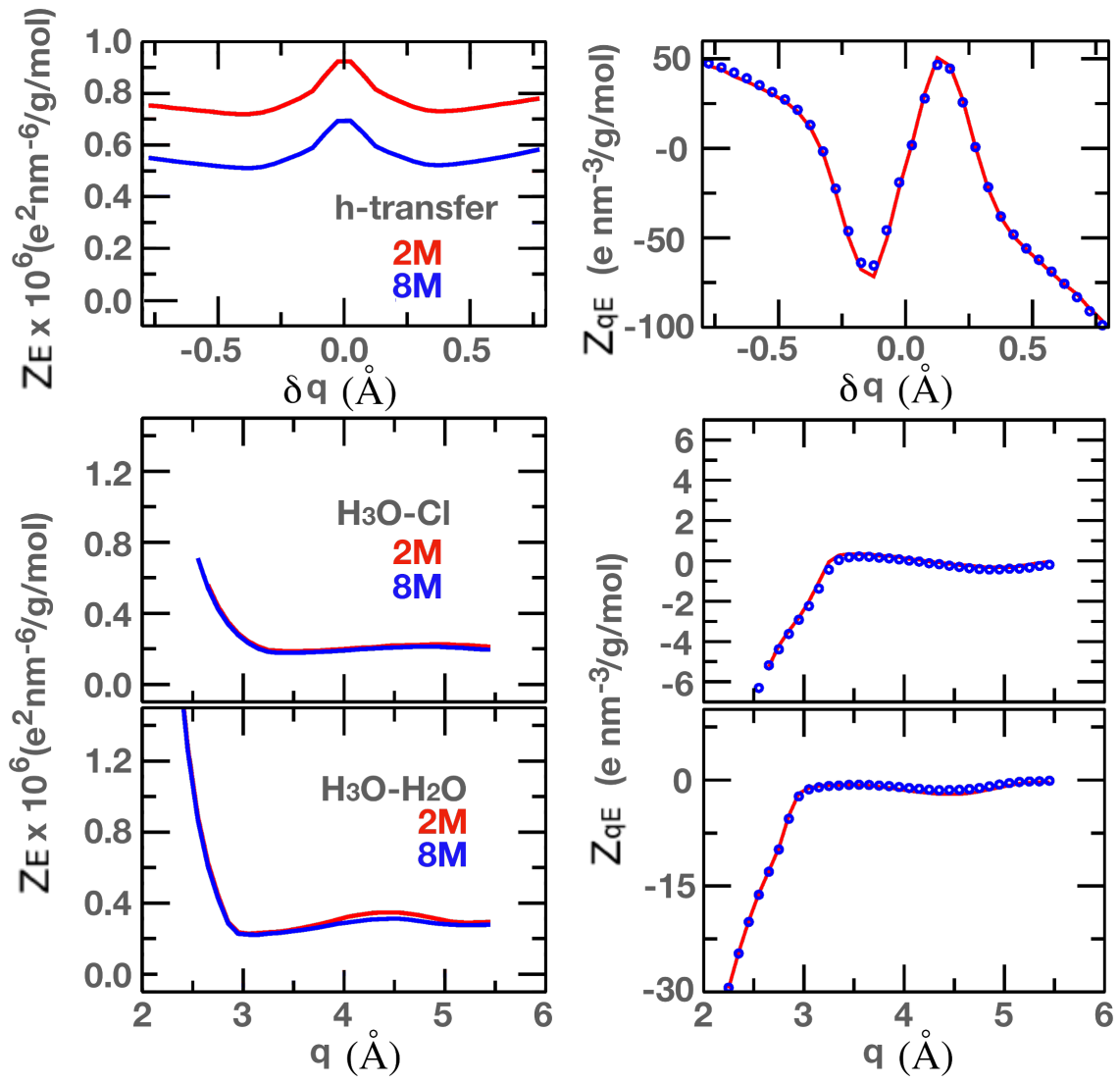

Figure S4: $Z_{E}$ and $Z_{q E}$ highlighting their dependency on the distance coordinate for the case of proton transfer (top), dissociation of ion pairs (middle), and water exchange (bottom). The right panel has the same color code as the left panel.

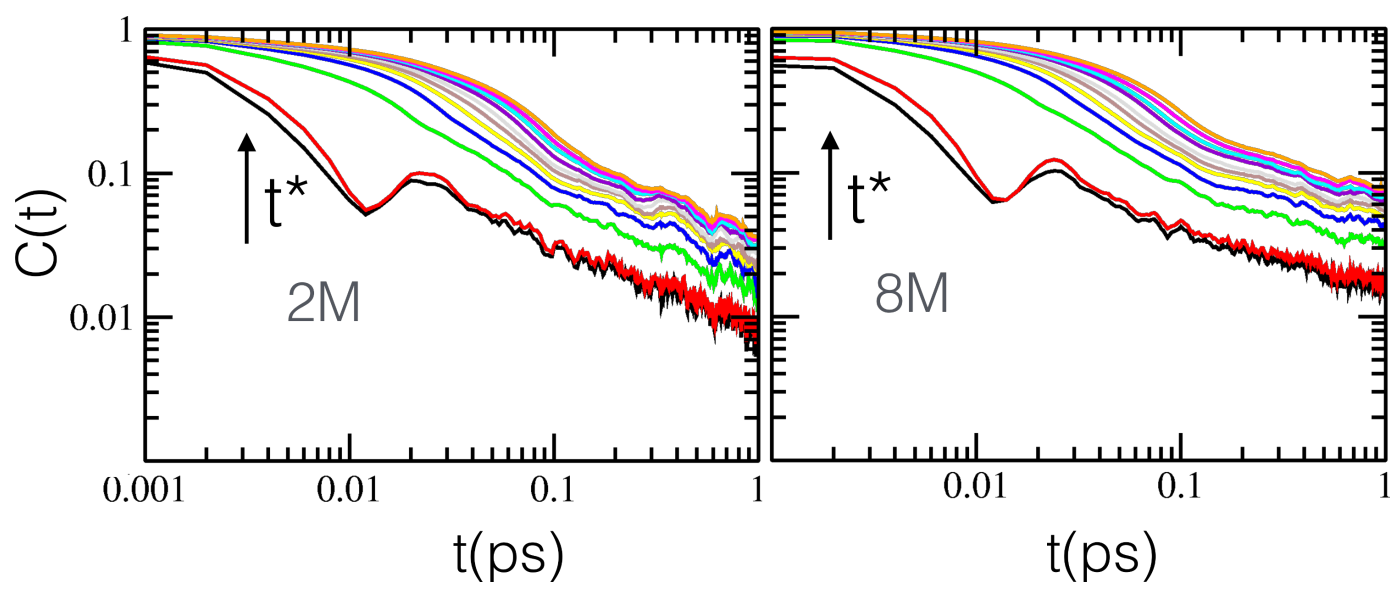

Figure S5: Normalized survival probability correlation function for the Zundel state at different $\mathrm{HCl}$ concentrations and at different $t^{\star}$. 